\title{
Oceanographic laboratories
}

\section{A. S. Laughton}

Oceanographic Institutions - Science Studies the Sea. By P. Limburg. Pp.265. (Elsevier/Nelson Books: New York, 1979.) $\$ 9.95$.

THE public image of a scientific laboratory and of the scientists who work in one is highly coloured by what is seen on television and by science fiction. It is therefore very valuable for a book to be published which explores the reality of research institutes and attempts to look at the motivation and day-to-day working of research scientists. By choosing a subject such as oceanography which has grown rapidly during the past three decades, but the roots of which go back to the last century, it is possible to relate the development of the science with the development of the laboratories studying it.

Limburg has written a book that tackles with varying success both the science and the laboratories. As a potted history of oceanography his first three chapters leave a good deal to be desired. There are inaccuracies (the first word in the acronym ASDIC is "Allied" not "Anti-"), a lack of understanding of some of the technology (the difference between reflection and refraction seismics) and a rather journalistic view of the development of scientific thought (from continental drift through stable continents towards plate tectonics). The historical review is very unbalanced. Two pages are devoted to diving and subsea habitats, whereas deepsea drilling is limited to 11 lines, and oceanography from space to six. Nearbottom technology scarcely gets a mention nor do the achievements of long-term deployments of instruments in the ocean, which depend crucially on the developments of marine acoustics.

The accounts given of the work in the major (and some minor) oceanographic laboratories in the USA are both informative and interesting, and the unravelling of the complexities of US Government agencies' funding of ocean research is revealing, if perhaps somewhat ephemeral. As the book proceeds, it becomes clearer and clearer that the author is really concerned with oceanography in the USA and I wonder why this was not made clearer in the title. Oceanography in all other countries is limited to one chapter and appears to include only those laboratories which the author was able to visit. No mention at all is made of oceanographic institutions in the USSR or in Japan. It cannot therefore be said, nor is it claimed in the Introduction, that the book is a comprehensive review of oceanographic institutions. What it does provide, and in this is its success, is a peep in through the doors of some of the laboratories, an insight into the enthusiasm and motivation of some oceanographers both great and small, and an indication of the breadth of the subjects studied.

For a student wishing to start a career in oceanography, especially in the USA, I warmly recommend this book.

A. S. Laughton, Director of the Institute of Oceanographic Sciences of NERC, has spent his working life in the field of marine geophysics, and has worked closely with several of the major oceanographic institutions in the USA.

\section{The wonder of it all}

\section{R.H. Dalitz}

Knowledge and Wonder: The Natural World as Man Knows It. Second edition. By Victor F. Weisskopf. Pp.312. (MIT Press: Cambridge, Massachusetts, and London, UK, 1980.) Hardback \$15, £9.30; paperback $\$ 5.90, £ 3.70$.

TODAY we have knowledge of our Universe covering distances from $10^{-16} \mathrm{~cm}$ to $10^{+28} \mathrm{~cm}$, and for time intervals from $10^{-24} \mathrm{~s}$ to $10^{+17} \mathrm{~s}$. All the phenomena observed within these limits are apparently governed by a very limited number of physical forces. The aim of this book is to lead non-scientists to appreciate the wonder of this situation, first by indicating simply how we know the facts and then by illustrating the wide variety of phenomena governed by these forces. Such an endeavour necessarily involves some oversimplifications, of course. Here the job is done in low key, showing how simple concepts based on the laws governing these forces can lead to an understanding of this strikingly wide variety of phenomena.

The book begins with space and time. It estimates the lifetimes of Earth and of the Universe, and the sizes and distances in the visible Universe. Two major forces are obvious to us from daily life, gravitation and electromagnetism. Their characteristics are outlined and related to the variety of phenomena to which they give rise. We explore the Solar System, understand the Milky Way as our galaxy, and relate it with far-off galaxies, made visible by telescopes. On Earth, we explore the structure of the atom and learn of its tiny nucleus, first deduced from Rutherford's alpha-particle scattering experiments. Both electrons and light show both particle and wave characteristics, and we soon reach quantum mechanics, with discrete energy levels for atoms, each level corresponding to a standing wave of electrons circulating about its atomic nucleus. The Coulomb force accounts for the binding of these circulating electrons to the nucleus, and also for the forces between atoms. We are shown the most common bonding mechanisms between atoms, and use the atoms as building blocks to make molecules, crystals and other solids and liquids, and so account for the materials around us as due to electromagnetic forces.

Next we are led to still shorter distances, to the nuclear and subnuclear worlds. The atomic nucleus consists of protons and neutrons, bound together by strong nuclear forces, and we soon understand why the stable nuclei are limited in number. An atom's chemistry then depends primarily on the proton number of its nucleus, and a pure chemical element can have various atomic mass values, corresponding to the number of neutrons in its nucleus. The ancient dream of transmuting other elements into gold is now seen to be possible, although so expensive that it has no financial value.

Recent experiments on the scattering of electrons and neutrinos by nuclei have shown that the nucleons themselves are composite, each containing three quarks. The rich spectroscopies of atoms and nuclei, each due to their multiplicity of component particles, are now paralleled by a spectroscopy of nucleons, due to excitation of the motions and spins of their component quarks. These experiments also show us that quarks, as well as electrons and neutrinos, have no detectable structure larger than $10^{-16} \mathrm{~cm}$.

We turn now to our terrestrial environment. Earth's long history is traced out, great changes occurring in its surface structure long before Man's emergence in recognizable form through evolutionary development. Thus, we come to the story of Life itself. The great steps are traced out, although not in detail, for we do not know them. We understand how the basic molecules needed to make up living material could form, perhaps through lightning flashes on the Earth's surface. The energy falling on Earth from the Sun was essential for the subsequent development of more complex organisms. Earth became covered in green, due to the chlorophyll which uses the energy reaching us from the Sun so efficiently. In the course of time, more complex organisms evolved, their structure being determined by the double-stranded DNA molecule, a thread carrying instructions for the construction of a living being, a thread replicated in its every cell. This evolution from simple to complex organisms, to living beings and to Homo sapiens, cannot be traced in detail but the general principles are outlined. The important role of mutations in this development is emphasized, and we see how the life on earth today could plausibly have 